\title{
AOR
}

Selected Papers of \#AolR2019:

The $20^{\text {th }}$ Annual Conference of the Association of Internet Researchers Brisbane, Australia / 2-5 October 2019

\section{CRITICAL SIMULATION: INVESTIGATING THE WORK OF MACHINE VISION IN VISUAL SOCIAL MEDIA CULTURE}

Nicholas Carah

School of Communication and Arts, The University of Queensland

Daniel Angus

Digital Media Research Centre, Queensland University of Technology

Adam Smith

School of Communication and Arts, The University of Queensland

\begin{abstract}
Social media are in the midst of an emphatic visual turn (Highfield \& Leaver 2016), characterised by the convergence of everyday visual expression with professional creative practice and advertising. The advertising-driven business models of social media platforms increasingly depend on automation. Platforms' use of machine vision is a key frontier in the algorithmic classification of culture. Machine vision algorithms automatically classify and misclassify faces, expressions, objects, and brand logos in the images users create and share. Images shared by platform users form vast databases used to train these same algorithms. Despite widespread use by social platforms, machine vision is poorly understood and accounted for in the study of everyday visual cultures. In this paper we detail a critical response to the use of automation in visual social media, called critical simulation. We outline a critical simulation framework, the 'Image Machine', focussed firstly on Instagram. The Image Machine comprises an Instagram data harvester, and open-access machine vision toolbox that allows digital humanities researchers to interrogate the inner workings of these algorithms and analyse their visual (mis)classifications. In this paper we showcase results from the Image Machine applied to images emanating from a major Australian music festival, Splendour in the Grass. This case examines not only how machine vision classifies and operates on culture, but also how these techniques are being operationalised within the advertising model and promotional culture of platforms like Instagram. We argue that the commercial application of machine vision is interdependent with the participatory culture of platforms like Instagram.
\end{abstract}




\section{Introduction}

The vernacular production of images of everyday life is integral to the commercial model of platforms like Instagram (Burgess 2015, Banet-Weiser 2012, Abidin 2016).

Participants do the everyday work of incorporating commercial experiences, object and logos into the stories they tell about themselves. A critical, and often under-examined, aspect of this relationship is that the everyday production of images is critical to training the machine vision classification of the platform itself.

As humans translate their lived experience into expressions, images and video on platforms, they are also building a vast set of algorithmic training data. That data is used to train and tune deep neural networks to recognise features and patterns in the images we create. The advertising-driven business models of visual social media platforms depend on automated, algorithmic processes, including the use of computational models to automatically classify images, a process called 'machine vision'. For machines, images are 'part of an operation' (Farocki 2004), raw material to be sorted and classified for defined ends (Andrejevic 2013). A t-shirt graphic might be linked to a brand name that can feed into an operation: for example, the delivery of an advertisement to a particular user. These machine vision systems both exploit and run counter to everyday cultural practices: for platforms, understanding images' meanings and representational qualities is not as important as learning how they can be used to nudge behaviour and shape cultural tastes (Packer 2013).

Despite widespread use by social media platforms, machine vision is poorly understood and accounted for in the study of everyday cultures; and machine vision techniques are yet to be embraced as part of the methodological repertoires of digital humanities. We contend that if Instagram is a key site in the 'biggest experiment of classification in human history' (Crawford 2018), then we must develop conceptual and methodological approaches for understanding its machine vision architecture and capacity to use machines to operate on, and organise, our cultural experience. Our response is to critically simulate the machine vision architectures of visual social platforms.

In the first instance, critical simulation involves building open-access software that use algorithms comparable to those used by the platform of interest. Critical simulation moves the digital humanities beyond employing computational tools to simply describe platform features or patterns of use, to also 'look under the hood' (Turow 2012). Once these algorithms are implemented, the next move in critical simulation is to examine how they intervene in culture, and how participants understand and creatively navigate cultural settings that are partly organised by machines. In the case of Instagram, bringing the inner workings and outputs of machine vision algorithmic decision making into dialogue with Instagram users allows us to better examine and reflect on how machines operate on and categorise everyday visual cultures (Carah \& Angus 2018). Critical simulation allows us to ask what kinds of new relationships are forming between vernacular practices and machine classification, including how everyday participation in visual social media also helps to train machine vision systems, and how human and nonhuman processes are interrelated in the production of digital culture. 


\section{Computational Critical Simulation Framework - The "Image Machine"}

The Image Machine is a critical simulation framework comprised of a number of modular software components that can be adapted to different visual social platforms, and machine vision architectures.

The Instagram data gathering module was developed in a manner that is not reliant on the use of Instagram's now deprecated platform-specific Application Programming Interface (API). This module allows the gathering of public images and metadata using hashtag, or geolocation queries. We use Puppeteer to interact with Instagram.com, intercepting and saving responses to requests that the page's JavaScript initiates, making it tolerant to regular changes to Instagram's web interface.

The Image Machine contains a machine vision sandbox that allows the integration of many standard 'supervised' and 'unsupervised' classification algorithms. Supervised approaches involve training algorithms to recognise pre-defined features, such as the presence of people, objects, logos, or text inside an image (see Figure 1). Unsupervised approaches involve training a machine to inductively recognise latent features in a data set, here these could be the use of colour, composition, or other distinctive image creation practices e.g. Instagram filters. Unsupervised approaches are crucial to developing a critical account of how machines determine patterns in cultural archives independent of human judgments about culture (Andrejevic 2013). Platforms like Instagram, Netflix and Spotify use unsupervised approaches to organise cultural material by identifying otherwise imperceptible connections, such as the nuanced beat structure and sonic topology of songs (Van den Oord et al. 2013).

The Image Machine can strip all unnecessary identifying information (e.g. user handles), as well as automatically blur faces in images for ethics purposes. Once gathered, images are processed by machine vision algorithms for analysis and interpretation. In Figure 1 the Detectron supervised algorithm, developed and used by Facebook throughout the mid-2010s ("Detectron," 2018), has identified bottles in an image returned using the query \#tgif. In such a configuration the Image Machine can mimic the fundamentals of platform approaches to machine vision, despite the smaller scale of data we are using to train our algorithms. 


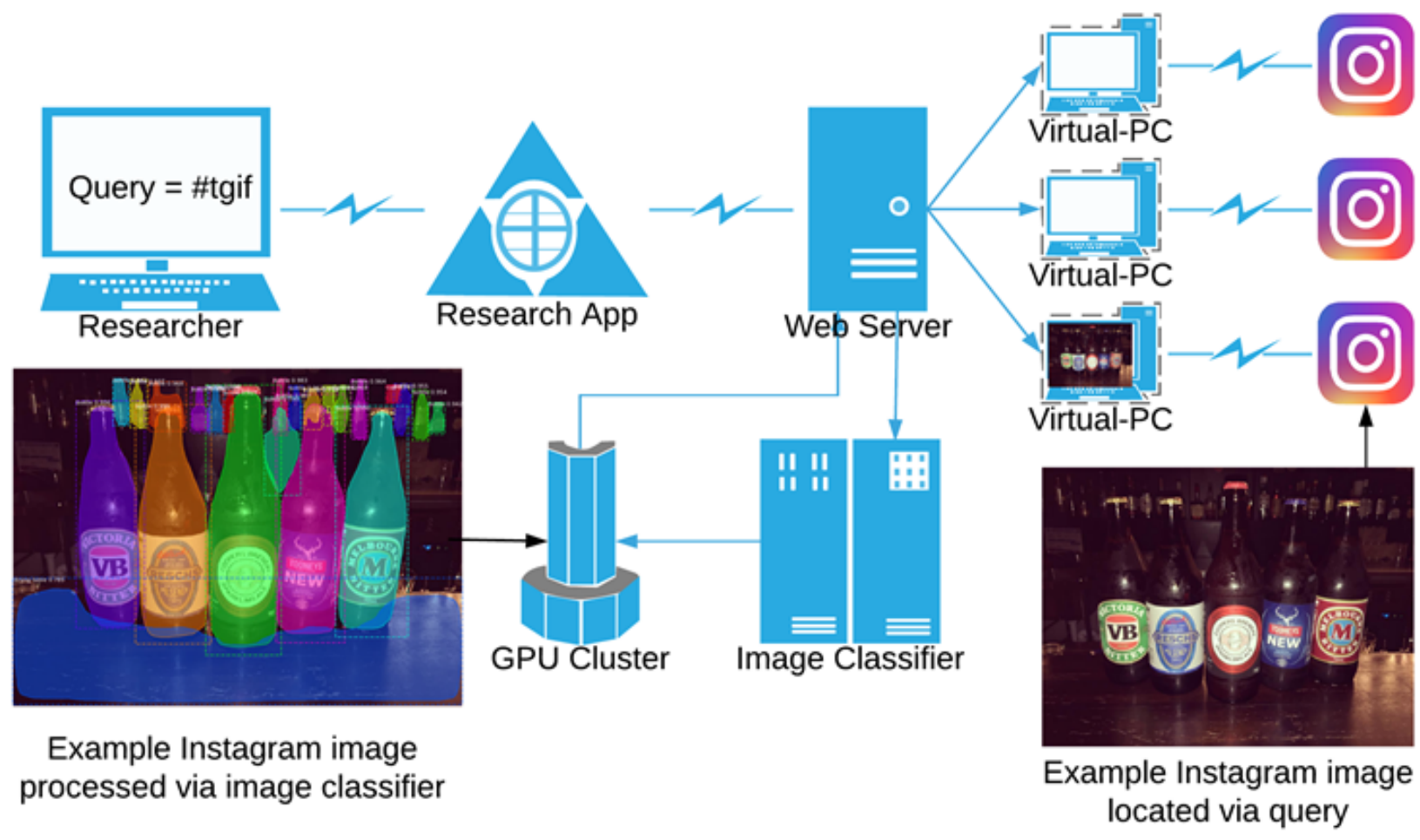

Figure 1: The Image Machine can gather and process images from Instagram to simulate how supervised machine vision can segment branded objects (e.g. brands of beer) within an image, each highlighted using a different coloured 'mask'.

\section{Results}

The first application of the Image Machine is to a major Australian music festival, Splendour in the Grass. The music festival Splendour in the Grass was selected as a temporally and geographically bound research site that features a combination of music performances, art installations, fashion, and brand activations. Each year, the festival also generates a large feed of images on Instagram, shared by the organisers, attendees, bands, artists, sponsoring brands and associated influencers. Brands build large interactive installations at the festival which, to use the terminology of marketers, are intended to be 'Instagrammable'. To road-test the Image Machine, we gathered 265,000 images posted to Instagram using hashtags associated with the festival, and then categorised them using both supervised and unsupervised classifiers. This case enables us to undertake a preliminary exploration of how not only how machine vision classifies and operates on culture, but also how these techniques are being operationalised within the advertising model and promotional culture of platforms like Instagram. We argue that the commercial application of machine vision is interdependent with the participatory culture of platforms like Instagram. 


\section{References}

Abidin, C. (2016). Visibility labour: Engaging with Influencers' fashion brands and \#OOTD advertorial campaigns on Instagram. Media International Australia 161(1): 86100.

Andrejevic, M. (2013). Infoglut: How too much information is changing the way we think and know. Routledge: London.

Banet-Weiser, S. (2012). AuthenticTM: The politics of ambivalence in a brand culture. New York: NYU Press.

Burgess, J. (2015). From 'Broadcast yourself' to 'Follow your interests': Making over social media. International Journal of Cultural Studies, 18(3): 281-285.

Carah, N., \& Angus, D. (2018). Algorithmic brand culture: participatory labour, machine learning and branding on social media. Media, Culture \& Society, 40(2), 178-194.

Crawford, K. (2018). 'Trouble with Bias' The 31st Annual Conference on Neural Information Processing Systems (NIPS 2017) Keynote Presentation. Retrieved from https://www.youtube.com/watch?v=ggzWlipKraM

Detectron. (2018, January 23). Retrieved from https://github.com/facebookresearch/Detectron

Farocki, H. (2004). Phantom Images. Public, 29.

Highfield, T., \& Leaver, T. (2016). Instagrammatics and digital methods: studying visual social media, from selfies and GIFs to memes and emoji. Communication Research and Practice, 2(1): 47-62.

Packer, J. (2013). Epistemology not ideology or why we need new Germans. Communication and Critical/Cultural Studies, 10(2-3), 295-300.

Turow, J. (2012). The daily you: How the new advertising industry is defining your identity and your worth. Yale University Press: New Haven.

Van den Oord, A., Dieleman, S., \& Schrauwen, B. (2013). Deep content-based music recommendation. In proceedings of Neural Information Processing Systems 2013 (NIPS 2013), 2643-2651. 